\title{
Modeling Approaches for Masonry Structures
}

\author{
D. Addessi ${ }^{1}$, S. Marfia ${ }^{2}$, E. Sacco ${ }^{*}, 2$ and J. Toti ${ }^{3}$

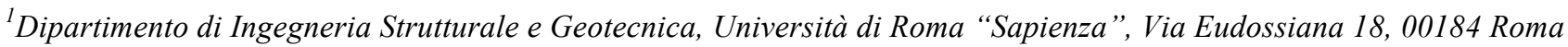 \\ Italy \\ ${ }^{2}$ Dipartimento di Ingegneria Civile e Meccanica, Università di Cassino e del Lazio Meridionale, Via G. Di Biasio 43, \\ 03043 Cassino Italy \\ ${ }^{3}$ Dipartimento di Ingegneria Civile, Edile-Architettura, Ambientale, Università dell' Aquila, Monteluco di Roio, 67040 \\ L'Aquila Italy
}

\begin{abstract}
Different scale approaches, micromechanical, multiscale and macromechanical or phenomenological, are presented to study the structural response of masonry elements. First, a micromechanical model is introduced and the masonry is considered to be a heterogeneous material, made of mortar and bricks joined by interfaces, where the mortarbrick decohesion mechanisms occur. To this end, a special interface model combining damage and friction is proposed.

Then, two multiscale procedures are presented, that consider regular arrangements of bricks and mortar, modeled by nonlinear constitutive laws which account for damage and friction effects. A homogenization technique is developed to derive two different equivalent continuum models at the macro-level, a micropolar Cosserat continuum and a nonlocal Cauchy model.

Finally, a macromechanical model, based on the adoption of a classical No-Tension Material (NTM) model, and on the presence of irreversible crushing strains, is proposed. A zero tensile strength is assumed, thus fracture strains arise when the stress is zero. Moreover, an elastoplastic model is considered for the material response in compression. Numerical applications are performed on a masonry arch and two masonry panels, by adopting the three approaches presented. Comparisons with experimental outcomes, published elsewhere, are performed.
\end{abstract}

Keywords: Masonry, Micromechanical Model, Multiscale approaches, Phenomenological Model.

\section{INTRODUCTION}

Masonry is a structural composite material, obtained by joining blocks or bricks of different nature and shape by means of mortar layers. The formulation of accurate and efficient procedures to evaluate the structural response of masonry constructions is still a challenging research field in civil engineering. Indeed, the determination of the safety state of historical and monumental masonry constructions subjected to seismic loading conditions is of great interest. The development of accurate stress analyses is fundamental not only to verify the stability of masonry constructions, such as old buildings, historical towns and monumental structures, but also to properly design effective strengthening and repairing interventions. Currently, the analysis of masonry structural response is a complex task. Masonry material is characterized by nonlinear mechanical behavior, even for low deformation levels, with anisotropy both in the linear and nonlinear range. Furthermore, masonry structures

*Address correspondence to this author at the Dipartimento di Ingegneria Civile e Meccanica, Università di Cassino e del Lazio, Meridionale, Via G. Di Biasio 43, 03043 Cassino Italy; Tel: +39 0776299 3659;

Fax:+390776 299 3392; Email: sacco@unicas.it often require $2 \mathrm{D}$ or $3 \mathrm{D}$ modeling approaches, i.e. more complex structural schemes compared with those usually used for concrete or steel framed structures.

To date particular focus has been given to the development of sophisticated numerical tools, often based on the Finite Element (FE) method, including 3D nonlinear constitutive laws. So far, nonlinear models implemented in suitable FE formulations appear to be the most promising. Several approaches have been proposed, differing with regard to the modeling scale used. Although other classification criteria can be adopted [1], in this study the focus is on three procedures that model masonry at micro, macro and micro-macro scales.

Micromechanical approaches are based on the distinct modeling of the brick and mortar joints, adopting different constitutive laws for each constituent. The mechanical properties of the bricks and mortar are obtained through experimental tests performed on each material. A drawback of this approach is that it requires a great computational effort. In a FE framework, both the unit bricks and mortar beds have to be discretized, resulting in a high number of nodal unknowns. In particular, the interface models have 
been proposed to simulate the mortar layers, when these present a reduced thickness, or to simulate the mortar-brick adhesion zones in masonry structures [2-9]. Indeed, the cracks occurring in masonry buildings are often located at the mortar joint-brick interfaces, which represent planes of weakness due to the coupling of two different materials.

Recently, in the case of masonry characterized by periodic or random texture, multiscale modeling is presented as a very promising approach. This matches the requirement of accurately reproducing microstructural mechanical and geometrical properties with the need to reduce the computational burden, with respect to a fully micromechanical analysis. The multiscale approach has been satisfactorily adopted in the structural analysis of periodic masonry. This is based on the analysis, at a lower level, of a Unit Cell (UC), containing all the information about the microstructure in the field of linear [10] and nonlinear [11] constitutive behavior, even accounting for viscoelastic effects $[12,13]$.

Two different Boundary Value Problems (BVP) are solved: one at the micro-scale level and the other at the macro-scale level. At the macro-scale, an equivalent homogeneous continuum is considered, whose formulation is completely stated except for the constitutive law, which is derived by solving the BVP formulated on the UC. At the micro-scale, all the constituents are modeled in detail, taking into account their geometrical arrangement, size and specific nonlinear constitutive laws. In this case, too, the mechanical properties of units and mortar joints are obtained through experimental tests. To connect the two scales, proper bridging conditions are formulated. At the micro-level, the classical Cauchy continuum is usually adopted, as the constituent constitutive laws are well established in this framework. On the other hand, different continuum models can be introduced at the macro-level. The standard first-order homogenization schemes are based on the use of the Cauchy medium also at macro-level. But, these models suffer from serious limitations when high deformation gradients occur, so that the macroscopic strain and stress fields considerably vary, and when strain-softening material behaviors are considered. To overcome these drawbacks, several techniques have been proposed, based on the use of interfaces [14] or nonlocal, higher-order and enriched micropolar models [15-22]. In particular, micropolar Cosserat models naturally introduce a material length scale into the constitutive description, to obtain a dependence of the overall response of the composite material on the absolute size of the constituents and to achieve a realistic description of the micro-structurally triggered macroscopic localization. Furthermore, also the mesh-dependency problems related to the strain-softening behaviors are overcome. Multiscale models in the framework of limit analysis [23-25] and of a stress approach [26] have also been proposed.

Macroscopic models (macro-models) are based on the use of phenomenological constitutive laws for the masonry material, considered as a homogenized medium. Masonry stress-strain relationships, adopted for the structural analysis, are derived by performing tests on masonry, without distinguishing between the bricks and the mortar behavior. A macroscopic model would be not suitable to describe accurately some of the micromechanisms occurring during the damage evolution, but this is very effective from a computational point of view. Several phenomenological models, based on friction-plasticity, damage or no-tension assumption have been proposed [27-31].

Here, the three different scale approaches described above are presented. First, a micromechanical model, considering the masonry as a heterogeneous material, made of mortar and bricks joined by means of interfaces, is illustrated. The computational strategy consists of modeling the brick units, the mortar joints and the interfaces responsible for the mortar-brick decohesion mechanisms and a special interface model, combining damage and friction, is adopted.

Then, two multiscale procedures are presented, modeling regular arrangements of bricks and mortar. Both multiscale procedures are based on the introduction of nonlinear constitutive laws for the bricks and the mortar, modeled as Cauchy media, combining damage and friction effects. A nonlinear homogenization technique is developed to identify two different equivalent continuum models at the macrolevel. One approach considers an equivalent Cosserat continuum at macro-level, which naturally regularizes the numerical response in the presence of damage. The other approach is based on the use of a nonlocal Cauchy continuum at macro-level, adopting the integral regularization technique. A comparison between the two multiscale approaches and experimental outcomes is provided.

Finally, a macro-model, based on the adoption of a phenomenological constitutive law for the masonry material is introduced. A generalization of the classical No-Tension Material (NTM) model accounting for irreversible crushing strains is proposed. A zero tensile strength is considered, thus fracture strains occur, when the stress assumes a zero value. Moreover, an elastoplastic model for the material response in compression is adopted, introducing a compressive finite strength. Thus, when the stress reaches the limit compressive value, plastic deformations develop.

\section{MICROMECHANICAL APPROACH}

A micromechanical approach to model masonry structures is illustrated. Masonry is considered as a heterogeneous medium, made of mortar and bricks joined by interfaces. Indeed, the cracks occurring in masonry are usually located at the mortar joint-brick interfaces, which represent planes of weakness due to the coupling of two different materials. This aspect has been investigated elsewhere through experimental tests, which demonstrated that decohesion between the masonry constituents is the main cause of its nonlinear response, e.g. [7].

The micromechanical computational strategy models separately the brick units, the mortar joints and the interfaces, where the mortar-brick decohesion mechanisms occur. Special interface models are introduced, taking into account the main nonlinear phenomena, such as damage, friction and inelastic slips. 
Below, the interface formulation modeling the clay brickmortar joint interaction is described in detail. Then, the accuracy and capability of the micromechanical approach in reproducing the experimental mechanical behavior of a masonry arch is tested and discussed.

\section{Interface Model}

The phenomenological interface model, proposed by Alfano and Sacco [8] and modified by Sacco and Toti [9], is presented. The micromechanical model includes interface damage, inelastic slips, friction and unilateral phenomena.

A local coordinate system $\left(0, x_{N}, x_{T}\right)$ is introduced at the typical point $\mathbf{x}^{\Im}$ on clay brick-mortar joint interface $\mathfrak{\Im}$, where the subscripts ${ }_{N}$ and ${ }_{T}$ indicate the normal and the tangential directions of the interface, respectively (Fig. (1a)). Denoting by $\mathbf{u}^{1}$ and $\mathbf{u}^{2}$ the displacement fields of the two bodies in adhesion, the relative displacement at the typical point $\mathbf{x}^{\mathfrak{S}}$ on $\mathfrak{I}$ is defined as $\mathbf{s}\left(\mathbf{x}^{\mathfrak{\Im}}\right)=\mathbf{u}^{2}\left(\mathbf{x}^{\mathfrak{\Im}}\right)-\mathbf{u}^{1}\left(\mathbf{x}^{\mathfrak{\Im}}\right)$.

At the point $\mathbf{x}^{\mathfrak{3}}$, a reference area is considered, which is representative of the response of the local behavior of the point $\mathbf{x}^{\Im}$ (Fig. (1b)). In other words, the overall response of the reference area gives the interface constitutive law. To derive this, the reference area is split in the undamaged part $A^{u}$, i.e., that not affected by the presence of microcracks, and the damaged part $A^{d}$, with microcracks. The ratio of the damaged area and the reference area is indicated with $D^{\Im}$, representing the interface damage at point $\mathbf{x}^{\Im}$. The damage parameter can vary from zero to one, with $D^{\Im}=0$ and $D^{\Im}=1$ corresponding to the undamaged state and to the totally damaged state, respectively. The stress-relative displacement relationship is defined in the undamaged and damaged parts of the reference area as:

$\boldsymbol{\sigma}^{u}=\mathbf{K s} \quad$ in $A^{u} ; \quad \boldsymbol{\sigma}^{d}=\mathbf{K}[\mathbf{s}-(\mathbf{c}+\mathbf{p})] \quad$ in $A^{d}$

where $\mathbf{K}$ is the interface stiffness matrix, $\mathbf{c}$ is the unilateral contact vector and $\mathbf{p}$ is the inelastic sliding vector. By averaging, the overall stress in the reference area is:

$\boldsymbol{\sigma}^{\Im}=\left(1-D^{\Im}\right) \boldsymbol{\sigma}^{u}+D^{\Im} \boldsymbol{\sigma}^{d}=\mathbf{K}\left[\mathbf{s}-D^{\Im}(\mathbf{c}+\mathbf{p})\right]$
In the local coordinate system, the stiffness matrix, the unilateral contact vector and the sliding friction vector are represented in components as:

$\mathbf{K}=\left[\begin{array}{cc}K_{N} & 0 \\ 0 & K_{T}\end{array}\right] \quad \mathbf{c}=H\left(s_{N}\right)\left\{\begin{array}{c}s_{N} \\ 0\end{array}\right\} \quad \mathbf{p}=\left\{\begin{array}{l}0 \\ p_{T}\end{array}\right\}$

where $H\left(s_{N}\right)$ denotes the Heaviside function, which assumes the following values: $H\left(s_{N}\right)=1$ if $s_{N} \geq 0$ and $H\left(s_{N}\right)=0$ if $s_{N}<0$.

To complete the interface model, the evolution of the interface damage $D^{\Im}$ and of the inelastic slip $\mathbf{p}$ have to be specified. Regarding the evolution of the damage parameter $D^{\Im}$, a model accounting for the coupling of fracture mode I and fracture mode II is considered. Indeed, the quantity $\eta_{k}=s_{k}^{0} / s_{k}^{f}$, defined as the ratio between the first cracking relative displacement, $s_{k}^{0}$, and the full damage relative displacement, $s_{k}^{f}$, where $k=N$ or $k=T$ in the normal or tangential direction, is introduced. Note that the ratio $\eta_{k}$ can be computed as function of the peak stress $\sigma_{k}^{0}$ and of the specific fracture energy $G_{c k}$ as $s_{k}^{f}=2 G_{c k} / \sigma_{k}^{0}$. Hence, the parameter $\boldsymbol{\eta}$, which relates the two fracture modes, is defined as:

$\eta=\frac{\left\langle s_{N}\right\rangle_{+}^{2}}{\|\tilde{\mathbf{s}}\|^{2}} \eta_{N}+\frac{s_{T}{ }^{2}}{\|\tilde{\mathbf{s}}\|^{2}} \eta_{T} \quad$ with $\tilde{\mathbf{s}}=\left\{\left\langle s_{N}\right\rangle_{+} s_{T}\right\}^{T}$

where the bracket operator $\langle\bullet\rangle_{+}$gives the positive part of the number. The equivalent relative displacement ratio is defined as:

$Y=\sqrt{Y_{N}^{2}+Y_{T}^{2}} \quad$ with $\quad Y_{N}=\frac{\left\langle s_{N}\right\rangle_{+}}{s_{N}^{0}} \quad Y_{T}=\frac{s_{T}}{s_{T}^{0}}$

The damage parameter is assumed to be a function of the relative displacement history, as follows:

$$
D^{\Im}=\max _{\text {history }}\left\{0, \min \left\{1, \tilde{D}^{\Im}\right\}\right\} \quad \text { with } \quad \tilde{D}^{\Im}=\frac{Y-1}{Y(1-\eta)}
$$

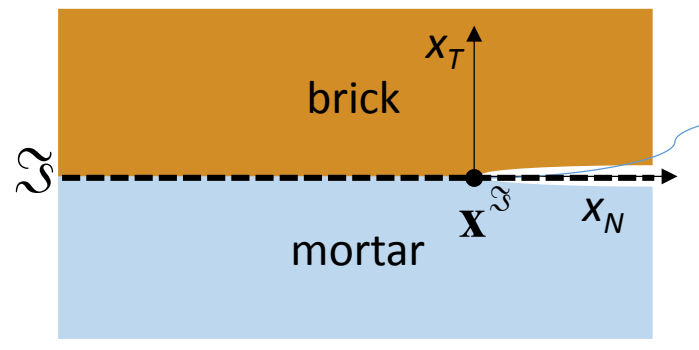

a)

Fig. (1). a) Brick-mortar interface; b) Reference area of interface. 
Note that, by using Equation (6), a linear stress - relative displacement relationship arises, when the ratio $s_{N} / s_{T}$ is assigned.

The evolution of the inelastic slip relative displacement is governed by the stress $\boldsymbol{\sigma}^{d}$ through the classical Coulomb yield function:

$\phi\left(\boldsymbol{\sigma}^{d}\right)=\mu\left\langle\sigma_{N}{ }^{d}\right\rangle_{-}+\left|\sigma_{T}^{d}\right|=\mu \sigma_{N}{ }^{d}+\left|\sigma_{T}^{d}\right|$

where $\mu$ is the friction coefficient and the symbol $\langle\bullet\rangle$ denotes the negative part of the number. The following nonassociated flow rule, together with Kuhn-Tucker conditions, is considered for the evolution of the vector $\mathbf{p}$ components:

$\dot{\mathbf{p}}=\dot{\lambda}\left\{\begin{array}{c}0 \\ \frac{d \phi}{d \sigma_{T}^{d}}\end{array}\right\}, \quad \dot{\lambda} \geq 0, \quad \phi\left(\boldsymbol{\sigma}^{d}\right) \leq 0, \quad \dot{\lambda} \phi\left(\boldsymbol{\sigma}^{d}\right)=0$

\section{Application: Masonry Arch}

The results of the micromechanical analysis of a masonry arch are presented. The simulations concern a masonry arch studied during the experimental campaign carried out by Cancelliere et al. [32]. This is made by alternating 23 standard clay bricks with 22 mixed mortars. The conventional numbering of bricks is shown in Fig. (2a). The geometrical data of the tested circular arch are the following: internal radius $r=456 \mathrm{~mm}$, width $w=255 \mathrm{~mm}$, thickness $t=120 \mathrm{~mm}$, height $f=510 \mathrm{~mm}$, abutment angle $\Phi=8^{\circ}$ and internal distance between the abutments $d_{a}=900 \mathrm{~mm}$. The tests performed in [32] investigate the mechanical response and the collapse mechanism of the masonry arch under the action of a vertical and eccentric point-wise force $F$. Specifically, the load is exerted by the action of a hydraulic jack on the extrados of the arch, precisely in correspondence of the $14^{\text {th }}$ clay brick (Fig. (2a)).

The developed FE model adopts, for the brick units and mortar joints, two-dimensional plane stress 4-node quadrilateral elements, and for the brick-mortar contact zone, 4-node interface elements Fig. (2b). Concerning the constitutive laws, the isotropic linear elastic model is used for both the clay-brick and mortar joints, while the nonlinear interface model describes the clay brick-mortar interaction.

The values of the mechanical properties of the brick and mortar are indicated in Table 1, based on the tests performed in [32]. The symbols $E$ and $\boldsymbol{v}$, reported in Table $\mathbf{1}$, denote Young's modulus and Poisson's ratio of the two masonry constituents, respectively.

The values of the interface parameters adopted in the numerical simulation are reported in Table 2 . By considering the interface zone as a composite material made from a thin layer of brick and a thin layer of mortar, the normal and shear stiffness are determined through the following homogenization procedure:

$$
K_{N}=\frac{E_{b} E_{m}}{h_{b} h_{m}\left(E_{b} / h_{b}+E_{m} / h_{m}\right)} \quad K_{T}=\frac{G_{b} G_{m}}{h_{b} h_{m}\left(G_{b} / h_{b}+G_{m} / h_{m}\right)}
$$

where $E_{b}, G_{b}$ and $E_{m}, G_{m}$ are the normal and shear moduli of the brick and mortar, respectively; the brick and mortar thickness are set $h_{b}=0.5 \mathrm{~mm}$ and $h_{m}=1 \mathrm{~mm}$, respectively. The evaluation of the interface strengths, $\sigma_{N}^{0}$ and $\sigma_{T}^{0}$, and interface fracture energies, $G_{c N}$ and $G_{c T}$, are determined on the basis of specific tensile and shear tests

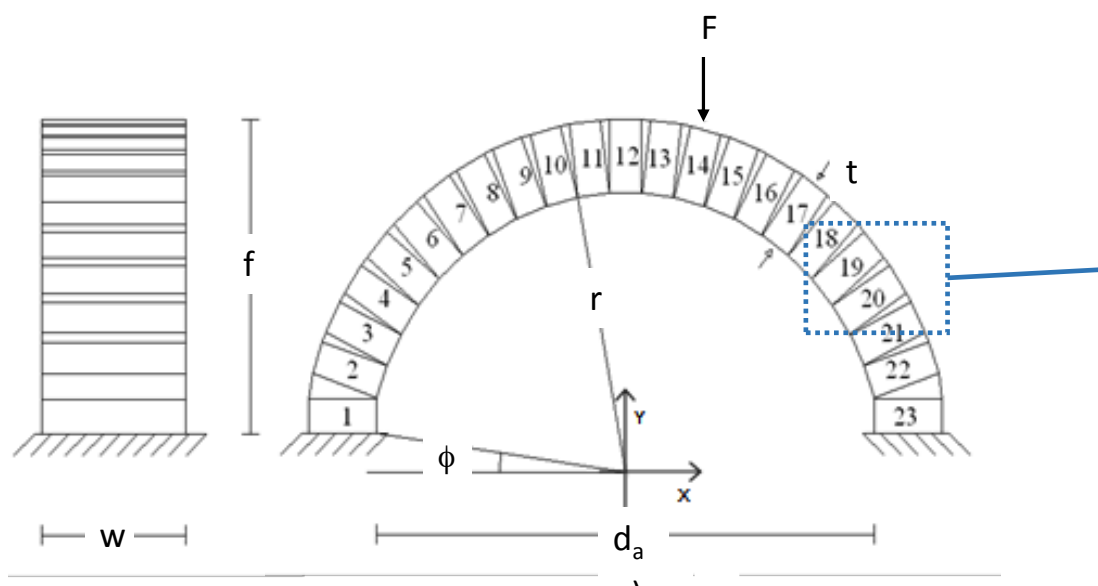

a)

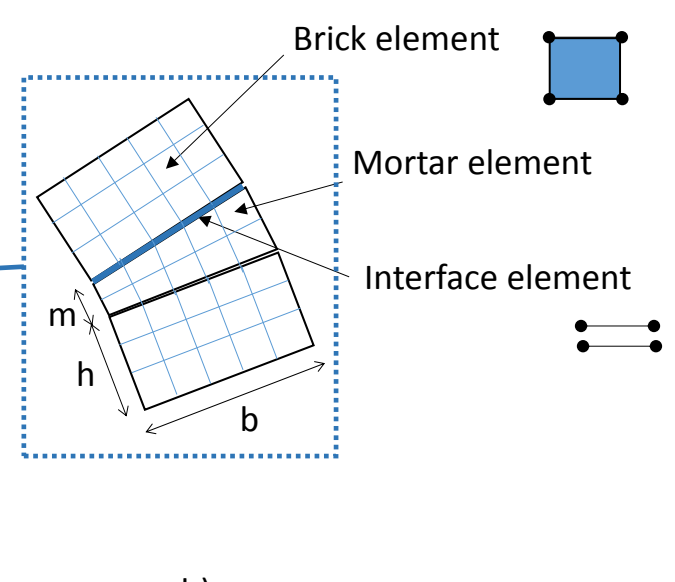

b)

Fig. (2). a) Geometry and boundary conditions; b) Detail of the finite element discretization.

Table 1. Mechanical properties of the masonry components.

\begin{tabular}{|c|c|c|}
\hline Material & $E\left[\mathbf{N} / \mathbf{m m}^{2}\right]$ & $\boldsymbol{V}$ \\
\hline Mortar & 1500 & 0.2 \\
\hline Clay-brick & 16000 & 0.2 \\
\hline
\end{tabular}


[32]. The friction coefficient can be determined also by a shear test and this typically varies from 0.5 to 1 .

Three different FE discretizations are adopted. These are obtained by introducing three discretization parameters, $n_{h}$, $n_{b}$ end $n_{m}$, indicating the number of subdivisions adopted for the height of the brick, the base of the brick and the thickness of the mortar joint, respectively Fig. (2b). In particular, the analyses are performed by setting the values of the mesh parameters $n_{h}=2$ and $n_{m}=1$, while three different values for the parameter $n_{b}$ are considered, $n_{b}=5,10$ and 20 .

In the numerical simulations, the action of the hydraulic jack is reproduced by applying at the central node at the top of the $14^{\text {th }}$ brick an incremental negative displacement $v$ along the $y$ direction of the reference system.

In Fig. (3a) the global response of the arch corresponding to the three adopted FE discretizations, plotted in terms of nodal reaction $F$ versus the incremental negative prescribed displacement $v$, is compared with the experimental data. The value of the failure load $(650 \mathrm{~N})$, deduced by applying the kinematic theorem of the limit analysis, is also reported. It can be emphasized that: the three numerical models satisfactorily reproduce the experimental behavior of the arch; the numerical result tends to the experimental curve by increasing the discretization along the base of the clay brick; the collapse load deduced by the limit analysis represents the upper bound of the ultimate strength of the arch.

Finally, Fig. (3b) shows the deformed configurations of the arch at $v=-0.08 \mathrm{~mm}$, for the adopted three discretizations. With reference to [32], it can be remarked that: the collapse mechanism is characterized by the formation of four hinges, two on intrados and two on extrados; the position of the brick-mortar interface, where the fracture opening occurs, is not influenced by the adopted mesh; the failure mechanism of the arch is mainly governed by the unilateral behavior of the mortar-brick interface.

\section{Multiscale Approach}

In the following, two multiscale models are developed in the framework of Cosserat and Cauchy theories [20, 22], to analyze 2D masonry structures, characterized by regular textures. A homogenization technique is adopted to derive the two different equivalent continua used to perform, at the macro-level, the masonry structural analysis. Hence, two BVPs are formulated at the macro- and micro-levels, respectively. Furthermore, the macro- and micro-levels are linked by a kinematic map and suitable boundary conditions are stated on the UC.

At the macro-level, the material point is denoted as $\mathbf{X}=\left\{X_{1}, X_{2}\right\}^{T}$, the displacement vector as $\hat{\mathbf{U}}$ and the associated strain as $\hat{\mathbf{E}}$.

\section{Micro-Level BVP}

Masonry is considered, at the micro-level, as a periodic composite material characterized by a regular distribution of bricks connected by horizontal and vertical mortar joints. In

Table 2. Interface properties.

\begin{tabular}{|c|c|c|c|c|c|c|}
\hline $\begin{array}{c}\sigma_{N}^{0} \\
{\left[\mathbf{N} / \mathbf{m m}^{2}\right]}\end{array}$ & $\begin{array}{c}G_{c N} \\
{[\mathbf{N} / \mathbf{m m}]}\end{array}$ & $\begin{array}{c}K_{N} \\
{\left[\mathbf{N} / \mathbf{m m}^{3}\right]}\end{array}$ & $\begin{array}{c}\sigma_{T}^{0} \\
{\left[\mathbf{N} / \mathbf{m m}^{2}\right]}\end{array}$ & $\begin{array}{c}G_{c T} \\
{[\mathbf{N} / \mathbf{m m}]}\end{array}$ & $\begin{array}{c}K_{T} \\
{\left[\mathbf{N} / \mathbf{m m}^{3}\right]}\end{array}$ \\
\hline 0.3 & 0.3 & 1500 & 3 & 0.3 & 750 \\
\hline
\end{tabular}
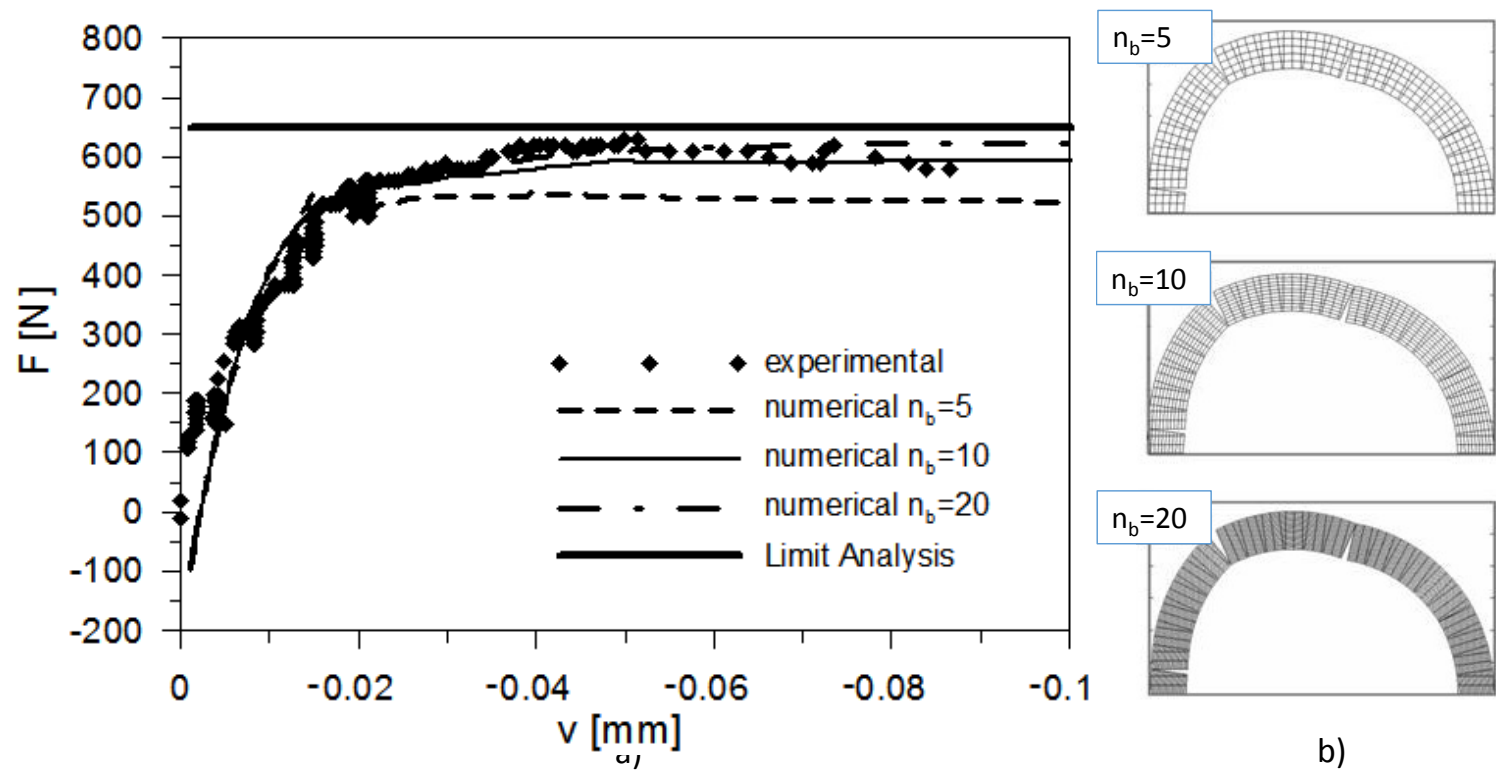

b)

Fig. (3). a) Comparison between the experimental and numerical results; b) Deformed configuration at $\mathrm{v}=-0.08 \mathrm{~mm}$. 
particular, the very common running-bond masonry texture is considered. The micromechanical analysis is performed on a Unit Cell (UC), properly selected at the micro-level to contain all the information on the geometry and texture of the constituents. The chosen rectangular UC, with dimensions $2 a_{1}$ and $2 a_{2}$, parallel to the coordinate axes $x_{1}$ and $x_{2}$, is reported in Fig. (4); the mortar thickness is denoted by $s$ and the brick sizes by $b$ and $h$. The classical Cauchy model is used at the micro-level, as most of the constitutive laws proposed for bricks and mortar are well stated in this framework.

To derive the constitutive response at each macroscopic material point $\mathbf{X}$, a BVP is solved on the UC shown in Fig. (4), whose center is located at $\mathbf{X}$.

The vector $\mathbf{u}=\left\{u_{1} u_{2}\right\}^{T}$ denotes the displacement field at each point $\mathbf{x}=\left\{x_{1} x_{2}\right\}^{T}$ of the UC domain $\omega$. The following representation form is assumed:

$\mathbf{u}(\mathbf{X}, \mathbf{x})=\mathbf{u}^{*}(\mathbf{X}, \mathbf{x})+\tilde{\mathbf{u}}(\mathbf{X}, \mathbf{x})$

where the displacement is expressed as the superposition of an assigned field $\mathbf{u}^{*}(\mathbf{X}, \mathbf{x})$, i.e., the kinematic map depending on the macro-level deformation vector $\hat{\mathbf{E}}$, and a perturbation field $\tilde{\mathbf{u}}(\mathbf{X}, \mathbf{x})$, which is unknown.

The kinematic map can be written in compact form as:

$\mathbf{u}^{*}(\mathbf{X}, \mathbf{x})=\mathbf{A}(\mathbf{x}) \hat{\mathbf{E}}(\mathbf{X})$

where $\mathbf{A}(\mathbf{x})$ is the kinematic map operator, which gives the displacement at point $\mathbf{x}$ of the UC in function of the macrostrain $\hat{\mathbf{E}}(\mathbf{X})$.

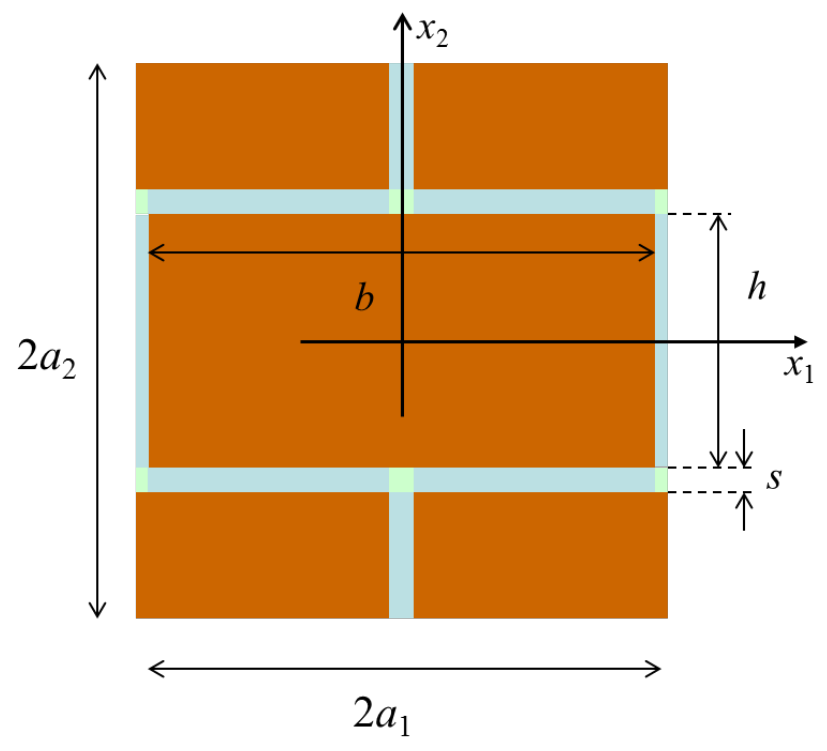

Fig. (4). Unit Cell (UC).
By applying the 2D Cauchy compatibility operator $\mathbf{d}$, the micro-level strain field $\varepsilon=\left\{\varepsilon_{11} \varepsilon_{22} \gamma_{12}\right\}^{T}$ is evaluated as:

$\boldsymbol{\varepsilon}=\mathbf{d} \mathbf{u} \quad$ in $\omega$

and results:

$\varepsilon=\varepsilon^{*}+\tilde{\varepsilon}$

where $\boldsymbol{\varepsilon}^{*}$ and $\tilde{\boldsymbol{\varepsilon}}$ are the strains associated to $\mathbf{u}^{*}$ and $\tilde{\mathbf{u}}$, respectively.

Considering the body forces as negligible at the UC level, the equilibrium equation results as:

$\mathbf{d}^{T} \boldsymbol{\sigma}=\mathbf{0} \quad$ in $\omega$

where the vector $\sigma=\left\{\sigma_{11} \sigma_{22} \tau_{12}\right\}^{T}$ contains the micro-level stress components. The BVP is completed by assuming suitable boundary conditions on the UC, which are different, when Cosserat or Cauchy continuum are considered at macro-level, as reported in detail in the following.

In what follows, nonlinear constitutive laws are adopted for the constituents at the micro-level. In particular, a damage-friction model is introduced for the mortar joints.

The constitutive law, accounting for the coupling of damage, unilateral contact and friction effects, is adopted for the mortar, herein modeled as a $2 \mathrm{D}$ continuum. The same local coordinate system as for the interface is used. The stress vector $\boldsymbol{\sigma}^{M}=\left\{\begin{array}{ccc}\sigma_{T}^{M} & \sigma_{N}^{M} & \tau_{N T}{ }^{M}\end{array}\right\}^{T}$, representing the stress in the mortar joint, is obtained by the relationship:

$\boldsymbol{\sigma}^{M}=(1-D) \boldsymbol{\sigma}^{u}+D \boldsymbol{\sigma}^{d}$

where $D$ denotes the damage variable and:

$$
\begin{aligned}
& \boldsymbol{\sigma}^{u}=\mathbf{C}^{M} \boldsymbol{\varepsilon}^{M} \\
& \boldsymbol{\sigma}^{d}=\mathbf{C}^{M}\left(\boldsymbol{\varepsilon}^{M}-\boldsymbol{\varepsilon}^{c}-\boldsymbol{\varepsilon}^{p}\right)
\end{aligned} \quad \text { with } \mathbf{C}^{M}=\left[\begin{array}{ccc}
C_{T T}^{M} & C_{T N}^{M} & 0 \\
C_{N T}^{M} & C_{N N}^{M} & 0 \\
0 & 0 & G^{M}
\end{array}\right]
$$

being $\boldsymbol{\varepsilon}^{M}=\left\{\begin{array}{lll}\varepsilon_{T}^{M} & \varepsilon_{N}^{M} & \gamma_{N T}{ }^{M}\end{array}\right\}^{T}$ the total strain vector and $\mathbf{C}^{M}$ the elasticity matrix of the mortar joint. The inelastic strain vectors $\boldsymbol{\varepsilon}^{c}$ and $\boldsymbol{\varepsilon}^{p}$, accounting for the unilateral contact effect and for the friction sliding, respectively, are defined as:

$$
\boldsymbol{\varepsilon}^{c}=H\left(\varepsilon_{N}\right)\left\{\begin{array}{lll}
\varepsilon_{T} & \varepsilon_{N} & \gamma_{N T}
\end{array}\right\}^{T} \quad \boldsymbol{\varepsilon}^{p}=\left\{\begin{array}{lll}
0 & 0 & \gamma_{N T}^{p}
\end{array}\right\}
$$

where $H\left(\varepsilon_{N}\right)$ is the Heaviside function.

The evolution of the inelastic slip strain component $\gamma_{N T}^{p}$ is governed by the classical Coulomb yield function. Furthermore, the evolution law adopted for the damage parameter $D$ is given by equation (6), where $Y$ and $\eta$ are now defined as: 
$Y=\sqrt{Y_{N}^{2}+Y_{N T}^{2}} \quad$ with $\quad Y_{N}=\frac{\left\langle\varepsilon_{N}^{M}\right\rangle_{+}}{\varepsilon_{N, o}} \quad Y_{N T}=\frac{\gamma_{N T}^{M}}{\gamma_{N T, o}}$

$\eta=\frac{1}{\alpha^{2}}\left[\left\langle\varepsilon_{N}^{M}\right\rangle_{+}^{2} \eta_{N}+\left(\gamma_{N T}^{M}\right)^{2} \eta_{N T}\right]$

$\alpha=\sqrt{\left\langle\varepsilon_{N}^{M}\right\rangle_{+}^{2}+\left(\gamma_{N T}^{M}\right)^{2}} \quad \eta_{N}=\frac{\varepsilon_{N, o} \sigma_{N, o}}{2 G_{c I}} \quad \eta_{N T}=\frac{\gamma_{N T, o} \tau_{N T, o}}{2 G_{c I I}}$

The two quantities $\varepsilon_{N, o}$ and $\gamma_{N T, o}$ are the first normal and tangential cracking strains, $\sigma_{N, o}$ and $\tau_{N T, o}$ are the peak values of the corresponding stresses, while $G_{c I}$ and $G_{c I I}$ are the fracture energies for mode I and mode II, respectively.

Taking into account the constitutive equations (16), formula (15) gives:

$\boldsymbol{\sigma}^{M}=\mathbf{C}^{M}\left(\boldsymbol{\varepsilon}^{M}-\boldsymbol{\pi}\right)$

where the total inelastic strain $\pi=D\left(\varepsilon^{c}+\varepsilon^{p}\right)$ is introduced.

To avoid strain and damage localization in the UC, a classical fracture energy regularization technique is used.

The linear elastic constitutive law is considered for the brick. Indeed, denoting by $\mathbf{C}^{B}$ the elasticity matrix of the brick, the stress-strain relationship is written in the form:

$\boldsymbol{\sigma}^{B}=\mathbf{C}^{B} \boldsymbol{\varepsilon}^{B}$

where

$$
\boldsymbol{\sigma}^{B}=\left\{\begin{array}{lll}
\sigma_{11}^{B} & \sigma_{22}^{B} & \tau_{12}^{B}
\end{array}\right\}^{T}
$$

and

$\boldsymbol{\varepsilon}^{B}=\left\{\begin{array}{lll}\varepsilon_{11}^{B} & \varepsilon_{22}^{B} & \gamma_{12}^{B}\end{array}\right\}^{T}$ are the stress and the strain vectors in the brick, respectively.

After determining the micro-level stress field $\boldsymbol{\sigma}$ in the mortar and in the brick, on the basis of the constitutive relationships presented above, the macroscopic stress components are computed using the Hill-Mandel macrohomogeneity condition. To this end, the virtual work evaluated at the macroscopic point is set equal to the average virtual work of the heterogeneous Cauchy medium in the UC.

\section{Cosserat-Cauchy Multiscale Model}

The variables and equations governing the Cosserat BVP at the macro-level are introduced. At each material point $\mathbf{X}$, the displacement vector is defined as $\hat{\mathbf{U}}=\left\{\mathbf{U}^{T} \Phi\right\}^{T}=\left\{U_{1} U_{2} \Phi\right\}^{T}$, containing three independent kinematic fields, i.e., the translations, $U_{1}$ and $U_{2}$, and the rotation, $\Phi$. Note that the vector $\mathbf{U}$ denotes the classical Cauchy displacement.
The compatibility equations, relating the deformation vector $\hat{\mathbf{E}}=\left\{\mathbf{E}^{T} \mathbf{K}^{T} \Theta\right\}^{T}$ to the displacement field $\hat{\mathbf{U}}$, are introduced in compact form as:

$$
\left\{\begin{array}{c}
\mathbf{E} \\
\mathbf{K} \\
\Theta
\end{array}\right\}=\left[\begin{array}{cc}
\mathbf{D} & \mathbf{0} \\
\mathbf{0} & \mathbf{D}_{K} \\
\mathbf{D}_{\Theta 1} & D_{\Theta 2}
\end{array}\right]\left\{\begin{array}{c}
\mathbf{U} \\
\boldsymbol{\Phi}
\end{array}\right\} \text { in } \Omega
$$

where $\mathbf{E}=\left\{\begin{array}{lll}E_{11} & E_{22} & \Gamma_{12}\end{array}\right\}^{T}$ contains the in-plane Cauchy strain components, $\mathbf{K}=\left\{K_{1} K_{2}\right\}^{T}$ collects the curvatures and $\Theta$ is the rotational deformation, measuring the relative rotation of the microstructure. The compatibility operators result as:

$\mathbf{D}=\left[\begin{array}{cc}\frac{\partial}{\partial X_{1}} & 0 \\ 0 & \frac{\partial}{\partial X_{2}} \\ \frac{\partial}{\partial X_{2}} & \frac{\partial}{\partial X_{1}}\end{array}\right], \quad \mathbf{D}_{K}=\left[\begin{array}{c}\frac{\partial}{\partial X_{1}} \\ \frac{\partial}{\partial X_{2}}\end{array}\right], \quad \mathbf{D}_{\Theta 1}=\left[\begin{array}{cc}\frac{\partial}{\partial X_{2}} & -\frac{\partial}{\partial X_{1}}\end{array}\right], \quad D_{\Theta 2}=1$

Accordingly, the vector $\hat{\Sigma}=\left\{\Sigma^{T} \mathbf{M}^{T} Z\right\}^{T}$, collecting the stress measures work conjugated with $\hat{\mathbf{E}}$ is introduced. The vector $\Sigma=\left\{\Sigma_{11} \Sigma_{22} \Sigma_{12}\right\}^{T}$ contains the classical in-plane Cauchy stress components, $\mathbf{M}=\left\{M_{1} M_{2}\right\}^{T}$ the couples and $Z$ is the stress variable conjugated with $\Theta$.

The equilibrium equations result as:

$$
\left[\begin{array}{ccc}
\mathbf{D}^{T} & \mathbf{0} & \mathbf{D}_{\Theta 1}{ }^{T} \\
\mathbf{0} & \mathbf{D}_{K}{ }^{T} & D_{\Theta 2}
\end{array}\right]\left\{\begin{array}{c}
\boldsymbol{\Sigma} \\
\mathbf{M} \\
Z
\end{array}\right\}=\left\{\begin{array}{l}
\mathbf{B} \\
C
\end{array}\right\} \text { in } \Omega
$$

the vector $\mathbf{B}$ containing the two body forces, $B_{1}$ and $B_{2}$, and $C$ being the body couple.

The displacement and traction boundary conditions complete the compatibility and equilibrium equations, governing the BVP at the macro-level:

$$
\hat{\mathbf{U}}=\hat{\mathbf{U}}_{0} \quad \text { on } \quad \partial \Omega_{U} \quad, \quad \mathbf{N} \hat{\boldsymbol{\Sigma}}=\hat{\mathbf{T}} \quad \text { on } \quad \partial \Omega_{T}
$$

where $\hat{\mathbf{U}}_{0}$ is the displacement vector prescribed on the boundary portion $\partial \Omega_{U}, \mathbf{N}$ is the matrix containing the unit vectors normal to the traction boundary contour $\partial \Omega_{T}$ and $\hat{\mathbf{T}}$ is the vector of the external tractions.

Once the BVP at the macro-level is defined, the kinematic map $\mathbf{A}(\mathbf{x})$ in equation (11) can be introduced. In particular, as proposed in [33] for 2D orthotropic homogenized media, it results: 


$$
\mathbf{A}(\mathbf{x})=\left[\begin{array}{lll}
\mathbf{A}^{1}(\mathbf{x}) \mathbf{A}^{2}(\mathbf{x}) \mathbf{A}^{3}(\mathbf{x})
\end{array}\right]
$$

with

$$
\begin{aligned}
& \mathbf{A}^{1}(\mathbf{x})=\left[\begin{array}{ccc}
x_{1} & 0 & \frac{1}{2} x_{2} \\
0 & x_{2} & \frac{1}{2} x_{1}
\end{array}\right] \\
& \mathbf{A}^{2}(\mathbf{x})=\left[\begin{array}{cc}
-\alpha_{1} x_{1} x_{2} & -\frac{1}{2} \alpha_{2}\left(x_{2}{ }^{2}+\lambda_{1} v_{12} x_{1}{ }^{2}\right) \\
\frac{1}{2} \alpha_{1}\left(x_{1}^{2}+v_{12} x_{2}{ }^{2}\right) & \alpha_{2} x_{1} x_{2}
\end{array}\right] \\
& \mathbf{A}^{3}(\mathbf{x})=\alpha_{3} s\left[\begin{array}{cc}
3 b_{1} x_{1}{ }^{2} x_{2}+c_{1} x_{2}^{3} \\
-3 b_{2} x_{1} x_{2}{ }^{2}-c_{2} x_{1}^{3}
\end{array}\right]
\end{aligned}
$$

where $\lambda_{1}=e_{2} / e_{1}$, while $e_{1}, e_{2}$ and $v_{12}$ are the Young's moduli and the Poisson ratio of the equivalent homogenized orthotropic material, respectively. Moreover, in formulas (28) it is set:

$$
\begin{gathered}
b_{1}=\lambda_{1}\left(1+\rho^{2} v_{12}\right), \quad c_{1}=b_{2}-2 \lambda_{2}, \\
b_{2}=\rho^{2}+\lambda_{1} v_{12}, \quad c_{2}=b_{1}-2 \rho^{2} \lambda_{2}, \\
S=\frac{10\left(1+\rho^{2}\right)}{a_{1}^{2}\left\{\lambda_{1}+\rho^{2}\left[\left(\lambda_{1} v_{12}-2 \lambda_{2}\right)\left(1+\rho^{2}\right)+\rho^{4}\right]\right\}}
\end{gathered}
$$

with $\lambda_{2}=e_{2} / g_{12}, g_{12}$ being the homogenized shear modulus and $\rho=a_{2} / a_{1}$.

Regarding the Cosserat-Cauchy approach, suitable BCs are enforced on the UC to reproduce the actual distribution of the perturbation field $\tilde{\mathbf{u}}$, according to those derived in [33]. These are schematically reported in Table 3 . In the first row, the referred macroscopic deformation components are reported; in the second row the $\mathrm{BCs}$ for the component $\tilde{u}_{1}$ along the horizontal and vertical edges of the UC are schematically reported, while in the third row those for the displacement component $\tilde{u}_{2}$ are shown. The symbol "p" indicates periodic BCs; "s" skew-periodic BCs, while " 0 " denotes zero perturbation displacement BCs.

\section{Cauchy-Cauchy Multiscale Model}

The variables and equations governing the Cauchy BVP at the macro-level are introduced. At each material point $\mathbf{X}$, the displacement vector is defined as $\hat{\mathbf{U}}=\mathbf{U}=\left\{U_{1} U_{2}\right\}^{T}$, containing only the translation components, $U_{1}$ and $U_{2}$. The associated compatible strain is $\hat{\mathbf{E}}=\mathbf{E}=\mathbf{D} \mathbf{U}$. Accordingly, the vector $\hat{\boldsymbol{\Sigma}}=\boldsymbol{\Sigma}$. The equilibrium equations result from (25):

$\mathbf{D}^{T} \boldsymbol{\Sigma}=\mathbf{B} \quad$ in $\quad \boldsymbol{\Omega}$

The displacement and traction boundary conditions completing the compatibility and equilibrium equations governing the BVP at the macro-level take a similar form as that reported in (26).

To overcome the problem due to the damage and strain localization, related to the strain-softening constitutive behavior, a nonlocal integral technique is used. In particular, the nonlocal macroscopic Cauchy deformation vector is defined as:

$$
\mathbf{E}_{n l}(\mathbf{X})=\frac{\int_{\Omega} \mathbf{E}(\mathbf{Y}) \psi(\mathbf{X}-\mathbf{Y}) d \Omega}{\int_{\Omega} \psi(\mathbf{X}-\mathbf{Y}) d \Omega}
$$

where $\mathbf{Y}$ is a typical point in $\Omega$ and $\psi$ is the standard Gaussian weight function, namely:

$\psi=\exp \left(-\frac{|\mathbf{X}-\mathbf{Y}|^{2}}{R^{2}}\right)$

\begin{tabular}{|c|c|c|c|c|c|c|c|c|c|c|}
\hline \multirow{4}{*}{$\tilde{u}_{1}$} & \multicolumn{2}{|c|}{$E_{11}$} & \multicolumn{2}{|c|}{$E_{: 22}$} & \multicolumn{2}{|c|}{$\Gamma_{12}$} & \multicolumn{2}{|c|}{$\Theta$} & $K_{1}$ & $K_{2}$ \\
\hline & & & & & & & & & & \\
\hline & $\mathrm{p}$ & $\mathrm{p}$ & $\mathrm{p}$ & $\mathrm{p}$ & $\mathrm{p}$ & & $\mathrm{p}$ & $\mathrm{p}$ & 0 & $\mathrm{p}$ \\
\hline & & & & & & & & & & \\
\hline \multirow{3}{*}{$\tilde{u}_{2}$} & & & & & & & & & & \\
\hline & $\mathrm{p}$ & $\mathrm{p}$ & $\mathrm{p}$ & 1 & $\mathrm{p}$ & 1 & $s$ & $s$ & $\mathrm{p}$ & $\mathbf{s}$ \\
\hline & & & & & & & & & & \\
\hline
\end{tabular}

with $R$ denoting the radius of the nonlocal domain at the structural level.

Once the BVP at the macro-level is defined, the kinematic map $\mathbf{A}(\mathbf{x})$ in equation (11) can be introduced. In particular, as proposed in [11] for 2D orthotropic homogenized media, it results $\mathbf{A}(\mathbf{x})=\mathbf{A}^{1}(\mathbf{x})$.

Table 3. BCs for the perturbation fields in the Cosserat-Cauchy homogenization. 


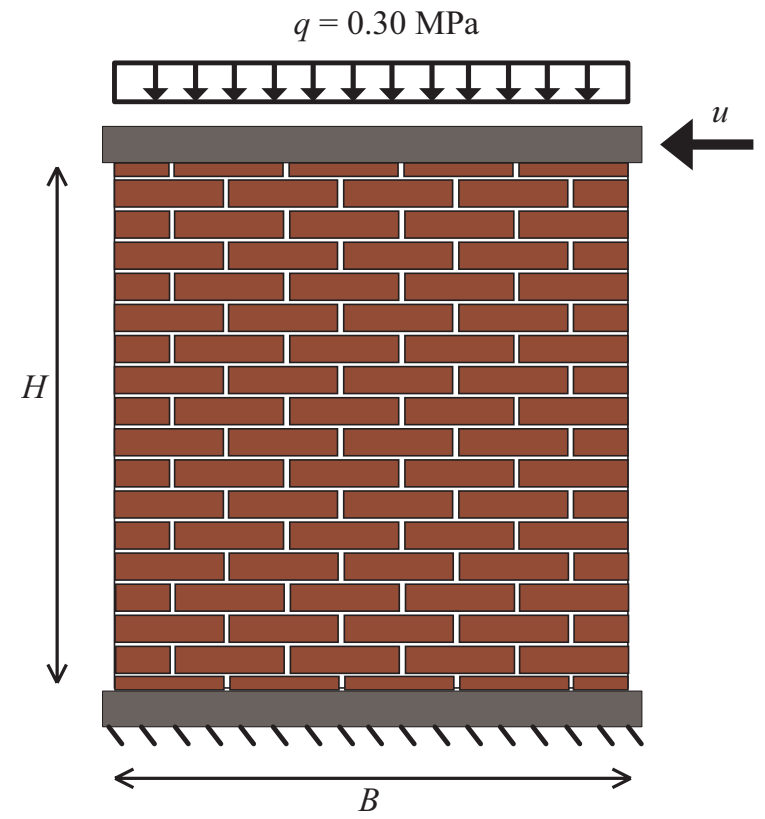

Fig. (5). Shear masonry wall: geometry and boundary conditions.

Concerning the Cauchy-Cauchy approach, periodic BCs are enforced on the UC to reproduce the actual distribution of the perturbation field $\tilde{\mathbf{u}}$.

\section{Numerical Application: Shear Masonry Wall}

The masonry wall shown in Fig. (5) is analyzed. This was studied experimentally by Raijmakers and Vermeltfoort [34]. The dimensions of the wall are: width $B=990 \mathrm{~mm}$, eight $H=1000 \mathrm{~mm}$, thickness $T=100 \mathrm{~mm}$. The wall is built with 18 courses of clay bricks, the first and last of which are clamped in steel beams. It is subjected initially to a vertical compressive load uniformly applied at the top side equal to $q=0.30 \mathrm{MPa}$.

During this phase the wall is completely restrained at the bottom side. Subsequently, the vertical translation and the rotation of the top side is restrained and a horizontal leftward displacement, monotonically increasing until the value of 4 $\mathrm{mm}$, is applied to these nodes. The geometrical parameters of bricks and mortar are the following: size of the brick $b=210 \mathrm{~mm}, h=52 \mathrm{~mm}$; thickness of the mortar joints $s=10 \mathrm{~mm}$. Furthermore, the material mechanical parameters are given in Table 4 , where $E$ and $\boldsymbol{v}$ are Young's and Poisson moduli, respectively. A 20x20 FE mesh is adopted for the computations. To model the top steel beam, 4-node quadrilateral FEs are used, assuming a linear elastic constitutive law with Young's modulus equal to

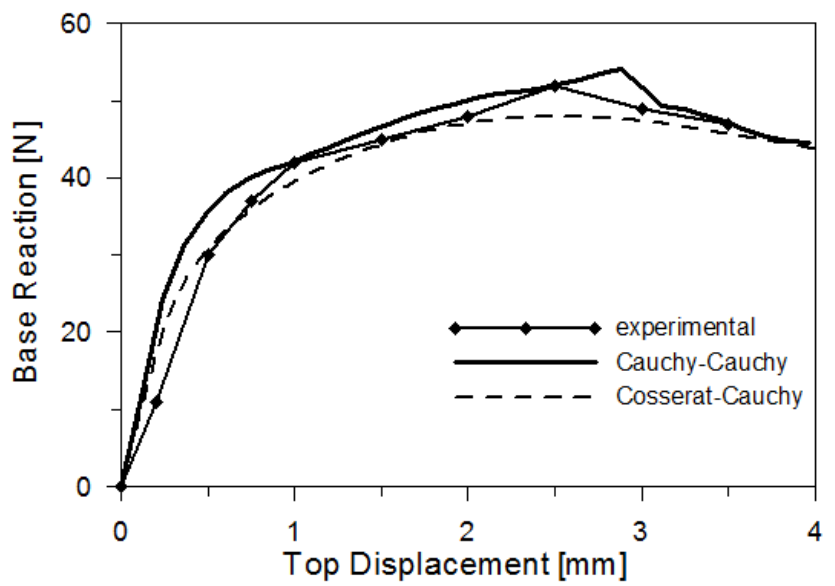

Fig. (6). Shearing masonry wall: global response curves.

$167000 \mathrm{MPa}$ and Poisson modulus 0.15.

In Fig. (6) the global response curve of the wall is reported, depicting the overall horizontal reaction computed at the bottom side versus the top applied displacement. Three different curves are shown, referring to the experimental outcomes (line with diamond symbols) and to the numerical results obtained by using the Cauchy-Cauchy (solid line) and Cosserat-Cauchy (dashed line) micro-macro approaches. It is evident that both the numerical curves match very well the experimental one. After the initial linear elastic behavior, the nonlinear mechanisms are activated. The global response curve reaches a peak load a little lower than that experimentally calculated in the case of the Cosserat-Cauchy model, at the applied displacement value of $2.5 \mathrm{~mm}$. In the case of the Cauchy-Cauchy approach, the peak load is a little higher than the experimental, and it is reached at a higher value of the applied displacement. Then, the global response curve shows a softening trend.

\section{MACROMECHANICAL APPROACH}

Masonry material constituting the structures of monumental and old constructions is often characterized by very low tensile strength with respect to the compression strength. Moreover, the tensile strength of the masonry can assume different values at different points of the construction and change during the life of the structure.

A realistic approximation for the evaluation of the mechanical response of the masonry material can be obtained assuming masonry as a no-tension material (NTM) $[35,36]$. Indeed, the collapse mechanisms of old masonry constructions are often characterized by the opening of cracks in tensile zones.

Table 4. Mechanical parameters of the shearing masonry wall.

\begin{tabular}{|c|c|c|c|c|c|c|c|}
\hline Material & $\begin{array}{c}E \\
{\left[\mathbf{N} / \mathbf{m m}^{2}\right]}\end{array}$ & $v$ & $\varepsilon_{N, o}$ & $\gamma_{N T, o}$ & $\begin{array}{c}G_{c l} \\
{[\mathbf{N} / \mathbf{m m}]}\end{array}$ & $\begin{array}{c}G_{c I} \\
{[\mathbf{N} / \mathbf{m m}]}\end{array}$ \\
\hline Mortar & 233 & 0.15 & 0.0015 & 0.004 & 0.00096 & 0.0057 \\
\hline Clay-brick & 1850 & 0.15 & & & & \\
\hline
\end{tabular}


The no-tension material (NTM) model is based on the fundamental hypothesis that the tensile strength is zero, while it considers a linear elastic behavior in compression. The no-tension model presents the following very special properties: a convex strain energy function governing the stress-strain relationship exists, thus the deformation process is reversible and there is no energy dissipation due to crack formation and evolution.

The no-tension material model is still a focus of much research on the behavior of old masonry structures. Several studies regard the NTM from a mechanical [37-39], mathematical [40] and computational point of view, developing displacement [41, 42], as well as stress and mixed variational formulations [43]. It has to be emphasized that, although the NTM is apparently simple, its numerical treatment is by no means so.

The assumption of the masonry linear elastic behavior in compression can be considered adequate only when the collapse mechanism is accompanied by very low compressive stresses. On the contrary, when the compression strength plays a significant role in the structural collapse load, the no-tension model does not appear to be suitable. This case may occur, for instance, for shear masonry panels and building walls [27-29].

Indeed, the crushing strain is irreversible during the whole loading history. As a matter of fact, the compression failure is affected by progressive damage and irreversible inelastic strains. To derive a simple but effective model, the inelastic behavior in compression is accounted for by developing a plasticity model, which ignores the damage and softening effects. The derived model results suitable for the description of the material crushing, when limited values of the compressive strain arise.

\section{The No-tension Plastic Model}

A two-dimensional plane stress elastoplastic NTM model is presented [29]. The strain $\mathbf{E}$ is partitioned into the sum of an elastic part $\mathbf{E}^{e}$ and two inelastic contributions $\mathbf{E}^{f}$ and $\mathbf{E}^{c}$, which account for fracture (in tension) and crushing (in compression), respectively:

$\mathbf{E}=\mathbf{E}^{e}+\mathbf{E}^{f}+\mathbf{E}^{c}$

A linear elastic relationship between the admissible stress $\boldsymbol{\Sigma}$ and the elastic strain $\mathbf{E}^{e}$ is assumed:

$$
\boldsymbol{\Sigma}=\mathbf{C} \mathbf{E}^{e} \quad \mathbf{C}=\frac{E}{1-v^{2}}\left[\begin{array}{ccc}
1 & v & 0 \\
v & 1 & 0 \\
0 & 0 & (1-v) / 2
\end{array}\right]
$$

where $\mathbf{C}$ is the isotropic elasticity matrix, $E$ and $v$ being the masonry Young's modulus and Poisson ratio.

Zero tensile strength is assumed. When the maximum normal stress reaches the zero strength value, an inelastic fracture strain occurs in the material. Denoting with $\Sigma_{1}$ and $\Sigma_{2}$ the principal values of the stress, the convex set $K$ of the admissible stresses is defined as:
$K=\left\{\Sigma: \quad \Sigma_{1} \leq 0, \quad \Sigma_{2} \leq 0\right\}$

The fracture strain tensor $\mathbf{E}^{f}$ is assumed to fulfill a normality rule with respect to $K$ :

$(\mathbf{S}-\boldsymbol{\Sigma})^{T} \mathbf{E}^{f} \leq 0, \quad \boldsymbol{\Sigma} \in K \quad \forall \mathbf{S} \in K$

Accordingly, the fracture strain $\mathbf{E}^{f}$ can be characterized as the solution of the following nonlinear problem:

$$
\mathbf{E}^{f}=\arg \min _{\mathbf{H} \in K^{*}}\left\{\frac{1}{2}\left(\mathbf{E}^{e c}-\mathbf{H}\right)^{T} \mathbf{C}\left(\mathbf{E}^{e c}-\mathbf{H}\right)\right\}
$$

with $\mathbf{E}^{e c}=\mathbf{E}^{e}+\mathbf{E}^{c}$ and $K^{*}$ is the polar cone of $K$. It can be proved that, for the isotropic case, the fracture strain $\mathbf{E}^{f}$, the strain $\mathbf{E}^{e c}$ and the stress $\boldsymbol{\Sigma}$ are coaxial, i.e. they present common principal directions [38].

Setting $E^{e c}{ }_{1} \leq E^{e c}{ }_{2}$, the fracture strain principal components are determined as:

$E^{e c}{ }_{1} \leq 0, \quad E^{e c}{ }_{2}+v E^{e c}{ }_{1} \leq 0$, no fracture is possible, so that the fracture principal strains result:

$E^{f}{ }_{1}=0 \quad E^{f}{ }_{2}=0$

$E^{e c}{ }_{1}>0$, the material is completely fractured and the fracture principal strains result:

$E^{f}{ }_{1}=E^{e c}{ }_{1} \quad E^{f}{ }_{2}=E^{e c}{ }_{2}$

$E^{e c}{ }_{1} \leq 0, \quad E^{e c}{ }_{2}+v E^{e c}{ }_{1}>0$, the material is fractured only in one direction, so that the fracture principal strains result:

$E^{f}{ }_{1}=0 \quad E^{f}{ }_{2}=E^{e c}{ }_{2}+v E^{e c}{ }_{1}$

The plastic yield function, based on the Drucker-Prager limit function, is written in terms of the principal stresses $\Sigma_{1}$ and $\Sigma_{2}$ as:

$F=\Sigma_{1}^{2}+\Sigma_{2}^{2}-\beta \Sigma_{1} \Sigma_{2}-\Sigma_{y}^{2} \leq 0$

where $\beta$ is a material parameter and $\Sigma_{y}$ is the initial yield limit value. The evolution law of the plastic strain is ruled by the equation:

$\dot{\mathbf{E}}^{c}=\dot{\lambda} \frac{\partial F}{\partial \boldsymbol{\Sigma}}$

with the Lagrange multiplier $\dot{\lambda}$ satisfying the loadingunloading and the consistency conditions:

$\dot{\lambda} \geq 0, \quad F \leq 0, \quad \dot{\lambda} F=0 \quad \dot{\lambda} \dot{F}=0$

In Fig. (7), the set of admissible stresses and the inelastic strain are schematically illustrated.

The no-tension plastic model is implemented in a numerical procedure based on the displacement FE formulation, using the backward Euler integration scheme and a return map algorithm. 


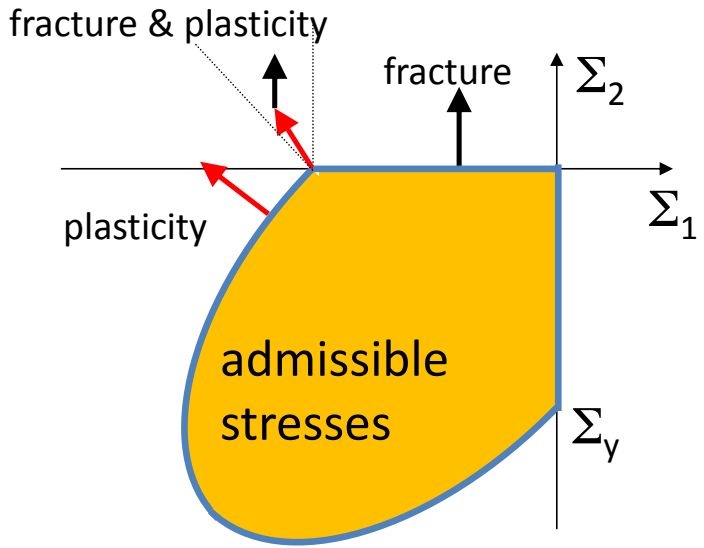

Fig. (7). No-tension plastic admissible stresses: normality rule of the fracture and crushing tensors.

\section{Numerical-Experimental Comparison}

A comparison between available experimental laboratory outcomes and the numerical results is reported, to verify the capability of the proposed no-tension plastic model to reproduce the behavior of masonry structural elements. One of the three masonry walls tested by Ganz and Thürlimann [44] and numerically analyzed by Lourenço [45], is analyzed. The geometry and the load applied on the masonry wall are schematically reported in Fig. (8). The following material properties are set:

$$
E=5460 \mathrm{MPa} \quad v=0.18 \quad \Sigma_{y}=7.6 \mathrm{MPa} \quad \beta=0.5
$$

The panel is subjected to an initial vertical force equal to $415 \mathrm{kN}$, distributed on the top of the wall; then, an increasing horizontal displacement on the top is prescribed.
Computations are carried out considering a 17x11 FE mesh made of 4-node quadrilateral elements.

In Fig. (9), the mechanical response of the shear panel, in terms of the horizontal force versus the horizontal displacement at the top, is reported in comparison with the experimental results obtained in [44] and with the numerical analyses developed in [45]. The very good agreement between the numerical results obtained using the developed no-tension plastic model and the experimental outcomes can be noted.

It can be emphasized that the illustrated no-tension plastic model is able to simulate the behavior of masonry structures, mainly made from ancient materials characterized by a very reduced tensile strength. The limited tensile constraint, together with the plasticity model, leads to a convex problem, which does not suffer from localization typically related to the strain-softening constitutive behavior. As a consequence, nonlocal stress-strain relations or other regularization techniques are not required. On the other hand, it is worthwhile noting that the proposed model could be successfully adopted for structural analyses, involving limited values of the compressive strains, so that the progressive material degradation and the softening behavior can be ignored. Thus, the proposed procedure can be adopted as a suitable tool for the design of restoring and reinforcement of old masonry structures.

\section{CONCLUDING REMARKS}

The modeling of masonry structures is an interesting and important task in the civil engineering field, related both to the safety of historic buildings and architectural heritage and to the safeguarding of people's lives. Several approaches were proposed, which can be classified in different ways. In this study, three widely adopted modeling strategies were

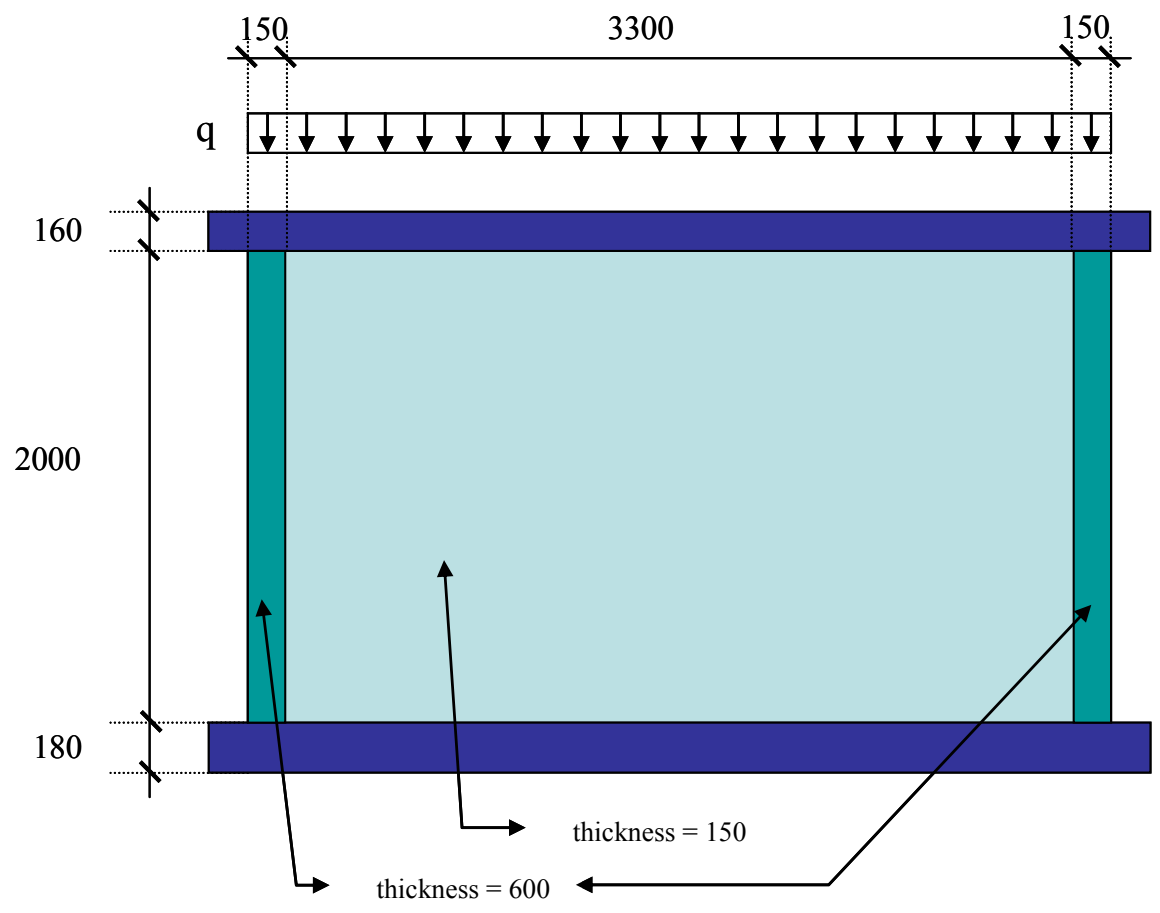

Fig. (8). Geometry and loads for ETH Zurich shear wall. 


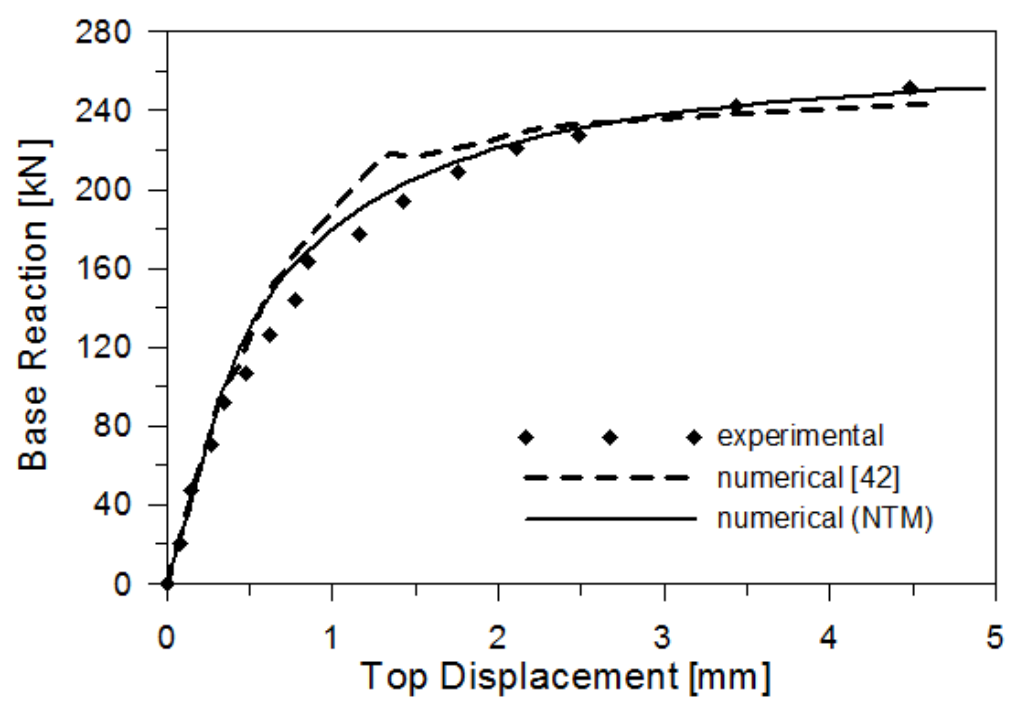

Fig. (9). Load - displacement curves for the ETH Zurich shear wall: comparison between experimental and numerical results.

presented, which differ in the scale at which the masonry structure is analyzed. In particular, a micromechanical, two multiscale and a macromechanical model were illustrated. Of course, these are not exhaustive, even if limiting interest only to the computational modeling techniques. Other classifications can be found, as well as other computational strategies. For instance, one of the most adopted for design purposes is the so-called macro-element approach, which was not considered here.

Comparing the presented modeling approaches on the basis of the accuracy in reproducing the masonry structural response, it emerges undoubtedly that the micromechanical model is the most precise. This is mainly true, when the focus is on the details of the local distributions of stresses and damage.

On the other hand, it clearly results that, when only the global aspects of the masonry behavior are investigated, more precisely, considering only the push-over response curves, all the approaches give satisfactory results. In this respect, it is worth noting that the good performances of the macromechanical model are strictly related to the correct identification of the phenomenological mechanical parameters, which is a difficult task. In the other two cases, i.e., in the micromechanical and multiscale approaches, this step is much simpler, as the mechanical parameters of the constituents are directly introduced. These are provided by laboratory or in-situ tests, or available from standard guidelines in the case of bricks and mortar.

The other relevant criterion, which has to be considered for the comparison of the modeling approaches, is based on the computational effort. Concerning this issue, the micromechanical approach is the most expensive, while multiscale models are characterized by a good compromise between accuracy, numerical efficiency and computational costs, particularly when parallel computing is used.

Hence, the choice of the most appropriate approach is strongly related to the specific typology of masonry structures to be analyzed and to the purposes of the analysis. For instance, the micromechanical approach can be satisfactorily adopted to reproduce the response of small size (laboratory) elements or of structures characterized by big bricks, where the size of the bricks has the same order of magnitude as the size of the structural element. Multiscale procedures can be efficiently adopted in the case of single structural elements. On the contrary, macromechanical, or even macro-element approaches, are more suitable in the case of large real buildings.

In conclusion, the choice of the modeling approach depends on the masonry construction typology, on the specific masonry behavior and on the available material parameters.

\section{CONFLICT OF INTEREST}

The authors confirm that this article content has no conflict of interest.

\section{ACKNOWLEDGEMENTS}

This research has been made possible due to the financial support of the project ReLUIS Consortium from the Italian Ministry of the Civil Protection.

\section{REFERENCES}

[1] P. Roca, M. Cervera, G. Gariup, and L. Pela', "Structural analysis of masonry historical constructions. Classical and advanced approaches," Arch. Comput. Methods Eng., vol. 17, pp. 299-325, 2010

[2] H.R. Lotfi, and P.B. Shing, "Interface model applied to fracture of masonry structures," J.Struct. Eng., vol. 120, no. 1, pp. 63-80, 1994.

[3] G. Giambanco, and L. Di Gati, "A cohesive interface model for the structural mechanics of block masonry," Mech. Res. Commun., vol. 24, no. 5, pp. 503-512, 1997.

[4] L. Gambarotta, and S. Lagomarsino, "Damage models for the seismic response of brick masonry shear walls. Part I: the mortar joint model and its applications," Earthquake Eng.Struct. Dyn., vol. 26, 1997.

[5] P.B. Lourenço, and J. Rots, "A multi-surface interface model for the analysis of masonry structures," J.Eng. Mech., ASCE, vol. 123 no. 7, pp. 660-668, 1997.

[6] D.V. Oliveira, and P.B. Lourenço, "Implementation and validation of a constitutive model for the cyclic behaviour of interface elements," Comput. Struct., vol. 82, pp. 1451-1461, 2004. 
[7] F. Fouchal, F. Lebon, and I. Titeux, "Contribution to the modelling of interfaces in masonry construction," Construct. Build. Mater., vol. 23, pp. 2428-2441, 2009.

[8] G. Alfano., and E. Sacco, "Combining interface damage and fiction in a cohesive-zone model," Int. J. Numer. Methods Eng., vol. 68, pp. 542-582, 2006.

[9] E. Sacco, and J. Toti, "Interface elements for the analysis of masonry structures," Int. J. Comput. Methods Eng. Sci. Mech., vol. 11, pp. 354-373, 2010.

[10] A. Anthoine, "Derivation of the in-plane elastic characteristics of masonry through homogenization theory," Int. J. Solids Struct., vol. 32, no. 2, pp. 137-163, 1995.

[11] E. Sacco, "A nonlinear homogenization procedure for periodic masonry," Eur. J. Mech. A/Solids, vol. 28, pp. 209-222, 2009.

[12] A. Cecchi, and A. Tralli, "A homogenized viscoelastic model for masonry structures," Int. J. Solids Struct., vol. 49, pp. 1485-1496, 2012.

[13] A. Taliercio, "Closed-form expressions for the macroscopic inplane elastic and creep coefficients of brick masonry," Int. J. Solids Struct., vol. 51, no. 17, pp. 2949-2963, 2014.

[14] G. Milani, "Simple homogenization model for the non-linear analysis of in-plane loaded masonry walls," Comput. Struct., vol. 89, no. 17-18, pp. 1586-1601, 2011.

[15] D. Addessi, E. Sacco, and A. Paolone, "Cosserat model for periodic masonry deduced by nonlinear homogenization," Eur. J.Mech. A/Solids, vol. 29, pp. 724-737, 2010.

[16] S. Brasile, R. Casciaro, and G. Formica, "Multilevel approach for brick masonry walls Part II: On the use of equivalent continua," Comput. Methods Appl. Mech. Eng., vol. 196, pp. 4801-4810, 2007.

[17] T.J. Massart, R.H.J. Peerlings, and M.G.D. Geers, “An enhanced multi-scale approach for masonry wall computations with localization of damage," Int. J. Numer. Methods Eng., vol. 69, no. 5, pp. 1022-1059, 2007.

[18] Stefanou, J. Sulem, and I. Vardoulakis, "Three-dimensional Cosserat homogenization of masonry structures: elasticity," Acta Geotech., vol. 3, no. 1, pp. 71-83, 2008.

[19] M.L. De Bellis, and D. Addessi, "A Cosserat based multi-scale model for masonry structures," Int. J. Comput. Eng., vol. 9, no. 5, pp. 543-563, 2011.

[20] D. Addessi, and E. Sacco, "A multi-scale enriched model for the analysis of masonry panels," "Int. J. Solids Struct., vol. 49, pp. 865-880, 2012.

[21] A. Bacigalupo, and L. Gambarotta, "Computational two-scale homogenization of periodic masonry: characteristic lengths and dispersive waves," Comput. Methods Appl. Mech. Eng., vol. 213, pp. 16-28, 2012.

[22] S. Marfia, and E. Sacco, "Multiscale damage contact-friction model for periodic masonry walls," Comput. Methods Appl. Mech. Eng., vol. 205-208, pp. 189-203, 2012.

[23] G. Milani, P.B. Lourenço, and A. Tralli, "Homogenization approach for the limit analysis of out-of-plane loaded masonry walls," J. Struct. Eng., vol. 132, pp. 1650-1663, 2006.

[24] G. Milani, P.B. Lourenço, and A. Tralli, "Homogenised limit analysis of masonry walls, Part I: Failure surfaces," Comput. Struct., vol. 84, pp. 166-180, 2006.

[25] G. Milani, P.B. Lourenço, and A. Tralli, "Homogenised limit analysis of masonry walls, Part II: Structural examples," Comput. Struct., vol. 84, pp. 181-195, 2006.
[26] S. Marfia, M. Ricamato, and E. Sacco, "Stress analysis of reinforced masonry arches," Int. J. Comput. Methods Eng. Sci., Mech., vol. 9, pp. 77-90, 2008.

[27] M. Lucchesi, C. Padovani, and N. Zani, "Masonry-like solids with bounded compressive strength," Int. J. Solids Struct., vol. 14, pp. 1961-1994, 1996.

[28] D. Addessi, S. Marfia, and E. Sacco, "A plastic nonlocal damage model," Comput. Methods Appl. Mech. Eng., vol. 191, pp. 12911310, 2002.

[29] S. Marfia, and E. Sacco, "Numerical procedure for elasto-plastic no-tension model," Int. J. Comput. Methods Eng. Sci. Mech., vol. 6, pp. $187-199,2005$

[30] L. Pelà, M. Cervera, and P. Roca, "Continuum damage model for orthotropic materials: Application to masonry," Comput. Methods Appl. Mech. Eng., vol. 200, pp. 917-930, 2011.

[31] D. Addessi, "A 2D Cosserat finite element based on a damageplastic model for brittle materials," Comput. Struct., vol. 135, pp. 20-31, 2014.

[32] I. Cancelliere, M. Imbimbo, and E. Sacco, "Experimental tests and numerical modeling of reinforced masonry arches," Eng. Struct., vol. 32, pp. 776-792, 2010

[33] D. Addessi, M.L. De Bellis, and E. Sacco, "Micromechanical analysis of heterogeneous materials subjected to overall Cosserat strains," Mech. Res. Commun., vol. 54, pp. 27-34, 2013.

[34] T.M.J. Raijmakers, and A.T. Vermeltfoort, "Deformation controlled tests in masonry shear walls, Report B-92-1156," TNOBouw, Delft, The Netherlands (in Dutch), 1992.

[35] J. Heyman, "The stone skeleton," Int. J. Solids Struct., vol. 2, pp. 249-279, 1966.

[36] J. Heyman, "The safety of masonry arches," Int. J. Mech. Sci., vol. 2, pp. 363-385, 1966.

[37] G. Romano, and M. Romano, "On the solution of structural problems with unilateral constitutive relations," Rendiconti Matematica Accad Lin, vol. 68, no. 8, pp. 104-113, 1979.

[38] G. Del Piero, "Constitutive equations and compatibility of external loads for linear elastic masonry-like materials," Meccanica, vol. 24, pp. $150-162,1989$

[39] M. Angelillo, "Constitutive relations for no-tension materials," Meccanica, vol. 28, no. 3, pp. 221-229, 1993.

[40] M. Giaquinta, and E. Giusti, "Researches on the equilibrium of masonry structures," Arch. Rational Mech. Anal., vol. 88, pp. 359$392,1988$.

[41] M. Lucchesi, C. Padovani, and A. Pagni, "A numerical method for solving equilibrium problems of masonry-like solids,". Meccanica, vol. 29, pp. 175-193, 1994.

[42] G. Alfano, L. Rosati, and N. Valoroso, "A numerical strategy for finite element analysis of no-tension material," Int. J. Numer. Methods Eng., vol. 48, pp. 317-350, 2000.

[43] M. Cuomo, and G. Ventura, "A complementary energy formulation of no-tension masonry-like solids," Comput. Methods Appl. Mech. Eng., vol. 189, pp. 313-339, 2000

[44] H.R. Ganz, and B. Thürlimann, "Tests on masonry walls under normal and shear loading," Report 7502-4, Institute of Structural Engineering, ETH Zurich, Zurich, Switzerland, 1984.

[45] P. B. Lourenço, "Computation Strategies for Masonry Structures, Phd Thesis,” Deft University Press: Netherland 1996. 